\title{
A Comparative Study of Automated PCB Defect Detection Algorithms and to Propose an Optimal Approach to Improve the Technique
}

\author{
Mohit Borthakur \\ Vishwakarma Institute of \\ Technology,Pune \\ A-Wing,Flat No.7,Punya Nagri \\ Society, Bibwewadi, Pune-37
}

\author{
Anagha Latne \\ Vishwakarma Institute of \\ Technology,Pune \\ 83 \& 84,H2-604,ManikMoti \\ Complex, Katraj, Pune-46
}

\author{
Pooja Kulkarni \\ Vishwakarma Institute of \\ Technology,Pune \\ 666, Upper Indira Nagar, \\ Bibwewadi, Pune. 37
}

\begin{abstract}
Automated visual printed circuit board (PCB) inspection is an approach used to counter difficulties occurred in manual inspection that can eliminate subjective aspects and then provide fast, quantitative, and dimensional assessments. Various concentrated work on detection of defects of printed circuit boards (PCBs) have been done, but it is also crucial to classify these defects in order to analyze and identify the root causes of the defects. However, besides the need to detect the defects, it is also essential to classify these defects so that the source of these defects can be identified. Based on studies done till now, some PCB defects can only exist in certain groups. Thus, it is obvious that the image processing algorithm could be improved by applying a segmentation exercise. This paper makes a comparative study of all such algorithms developed till date, to analyze their shortcomings and thereby provide an optimal approach to detect maximum of the defects with higher accuracy as well as with speed. This approach uses morphological image segmentation algorithm and simple image processing theories. The given algorithm can overcome most of the defects of previous algorithms and detect more than $80 \%$ of defects in a given PCB which ranges from missing components, broken tracks, misplaced components etc.
\end{abstract}

\section{General Terms}

Image Processing, Pattern Recognition, Algorithm, MATLAB

\section{Keywords}

PCB Testing, Digital Image Processing, Morphological Operators, Pattern Classification, Wavelet Transform

\section{INTRODUCTION}

The PCB (Printed Circuit Board) industry continues to adopt increasingly higher Windowing and Defect Extraction Operation levels of integration and achieving higher and higher levels of component density. As a consequence, the tolerance on PCB assembly has become tighter and tighter. This causes an increased need for reliable and accurate visual inspection of PCB boards. The manufacturing of PCB circuits uses SMT (Surface Mount Technology). The SMT circuit assembly consists of three major processes, screen printing solder paste on the PCB, component placement and then solder re-flow in a convection oven. Correspondingly, there are three main tasks of vision inspection in PCB assembly:
1. Solder Paste Inspection
2. Component Placement
3. Post-reflow Inspection

Placing a proper amount of solder paste on a pad is the key to prevent unwanted opens or shorts. Sometimes, it is possible to catch these unwanted opens or shorts using an in-circuit-test after all components are placed on the board, but most solder paste defects are impossible to catch after components are mounted.

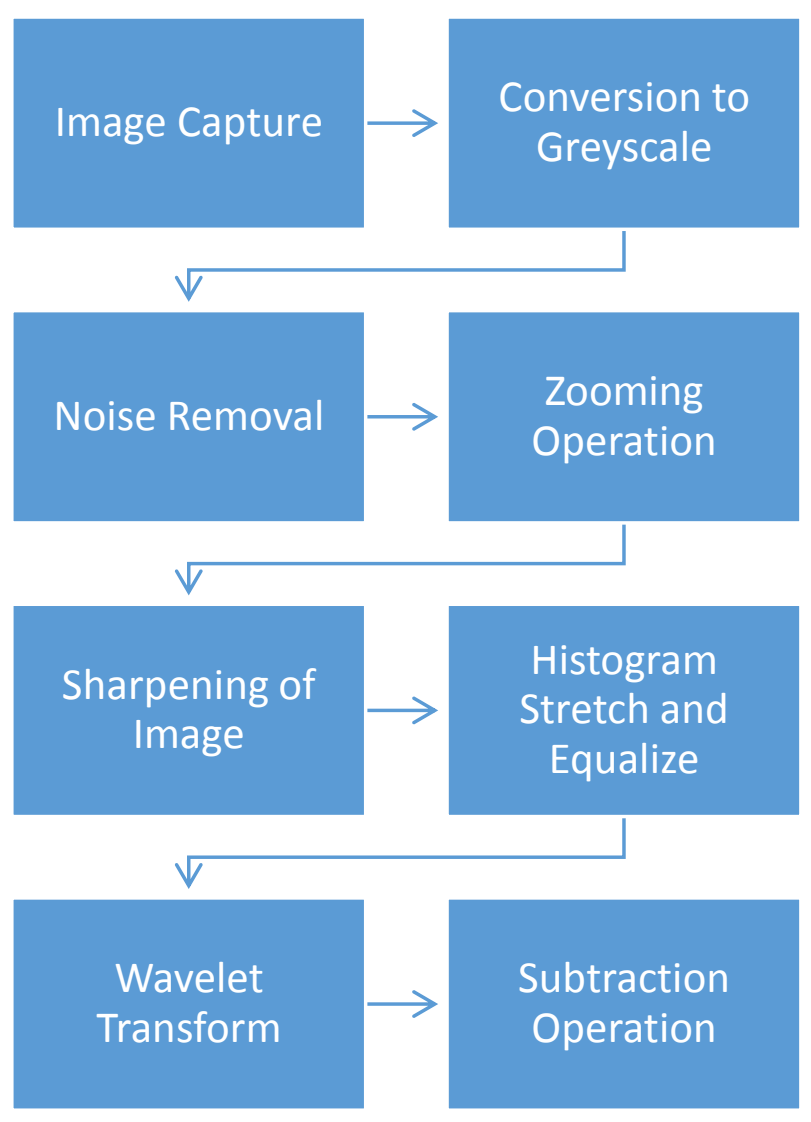

Fig 1.Basic Block Diagram of Proposed System

Image difference operation is frequently used in automated printed circuit board (PCB) inspection system as well as in many other image processing applications. The inspection system performance depends critically on the speed of this operation, which is a common problem related to the image difference. In many of the cases thresholding operation is performed before difference operator is used in order to extract out certain features. The flow of the operation is depicted in Fig 1. 


\section{BACKGROUND}

Moganti proposed [1] three categories of automated PCB inspection technique: referential approach, rule-based approach and hybrid approach [Fig 2].The referential approach compares the test PCB image against the reference PCB image. There are two major techniques: image comparison and model-based technique. Image comparison, which is the simplest technique, consists of comparing both images pixel-by-pixel [2] by XOR logic operator. The operation is also called as image difference operation. It is based on the assumption that, any difference between the reference and the test image is considered as a defect.

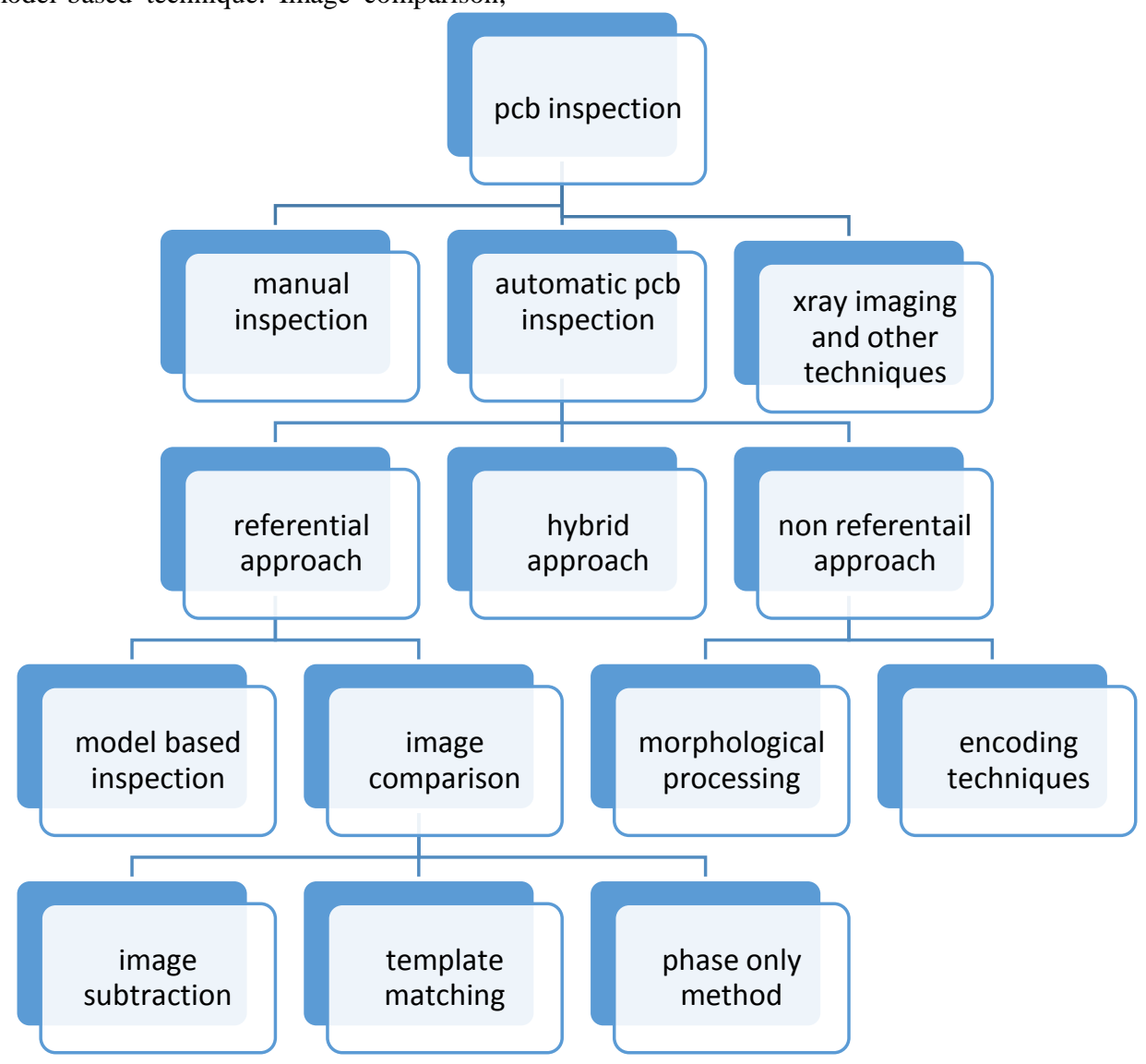

Fig 2. Classification of Detection techniques

The operation is simple but the main difficulty is to determine a precise alignment of the reference image and the test image, which makes its utilization difficult. More sophisticated

technique involves feature matching but this method normally requires a large number of templates.

Model-based methods are techniques, which match the pattern under inspection with a set of predefined models. Numerous techniques were proposed under model-based methods. They are tree representation technique [3], connectivity based technique [4], N-tuple technique [5] and Run Length Encoding (RLE) [6-8] based technique. RLE based technique is the best technique among the other model-based methods in the sense that it is possible to not just detect but also locate the defects. Under this technique, a PCB image is compressed in RLE data and the image difference operation is done on compressed RLE data. Rule-based approaches on the other hand test and determine whether each feature of PCB image falls within the required dimensions or not. These methods typically use morphological techniques like erosion and dilation as basic operation [9-11]. It does not require a reference model.

The hybrid approach is a combination of the reference-based and non-reference approaches. It has the advantages of these two approaches. However the approaches may be too complex and result in high computational costs. Among the numerous approaches that have been proposed in the literature so far, model-based referential approach has been preferred. Ercal et. al. [6] claimed that RLE-based technique is an effective way to minimize the storage of data. However, from time to time, the hard disk and memory (RAM and ROM) becomes cheaper and cheaper, the limitation of storage data is not a serious problem anymore. It is also claimed that RLE-based technique can be used to reduce the inspection time of a PCB inspection system. However, simulation result shows that it takes about 6.399 seconds to convert a $400 \times 400$ pixels Bitmap image into RLE data. The simulation is done using MATLAB.

The simulation result indicates that the bitmap to RLE conversion consumes a lot of time. Similarly for full resolution image difference operation, the image is needed to be scanned pixel-by-pixel in order to identify and to count the presence of pixel value zero representing object pixels. That is the reason why the image difference operation and the bitmapto-RLE conversion individually have almost the same processing time. Furthermore, the RLE data is needed to reconstruct back into bitmap image for visualization of defects after the systolic image difference algorithm on RLE data is done. To solve the shortcoming, a segmentation algorithm to divide a PCB images into basic patterns. The segmentation 
algorithm is essential to facilitate the distribution of image data in parallel processing for faster inspection response.

The computation of the wavelet transform of a twodimensional signal, i.e. image, is applied as a successive convolution by a filter of row/column followed by a column/row. The input image can be divided into 4 parts: approximation (LL1), horizontal detail (LH1), vertical detail (HL1) and diagonal detail (HH1) [12] where the size of each part is half compared to the input image. Again, same iteration is applied to the remaining approximation. Instead of the parallel processing of the RLE-based image difference operation, there is still a quadrant for the inspection of the printed circuit board (PCB). This technique using wavelet transform can be treated as image-to-image transformation.

\section{METHODOLOGY}

\subsection{Shortcomings of Previous Algorithms}

3.1.1 The basic automated defect detection starts with morphological operations on bare or mounted PCB. Few of such operations performed on PCBs are:

Erosion: It reduces the number of pixels from the boundary. The removal of number of pixels depends upon size of the structuring element. Erosion has the effect of deleting spiky edges. The spikes at the edge are removed and the track looks more superior and hence the detection of defects in the printed circuit board becomes easy.

Dilation: It adds pixels to the boundaries of the images. The number of pixels added depends on the size and shape of the structuring element. Dilation has the effect of filling in the valleys between spikes edges. The spiky edges at the corners and as well between the tracks are filled, so that the gaps or bites in the tracks are removed.

Opening: It generally smoothens the contours of the image, breaks down narrow bridges and eliminates thin protrusions. Opening operation connects the tracks that have undergone defects like burning or improper itching.

Closing: This operation generally tends to fuse up narrow breaks and small holes. It is an operation in which it separates or removes the unwanted path. Thus the tracks get separated from each other. It helps to detect those track defects, the presence of which is not wanted and may lead to short circuits.

Boundary Extraction: It is used to extract the boundaries of the given image depending on the size of structuring element used. . Using this operation one can get an idea about the boundary of tracks and the size of the circuit with respect to tracks.

As can be seen above, the basic process of defect detection using morphological operations can be implemented only to detect the defects in tracks. So on a basic level it is successful, but once mounted PCBs are used and defects are to be detected, then it fails.

3.1.2 The next best approach that has been used so far is the image subtraction operation using XOR operator [Fig 3].This process considers pixel by pixel value of the image and compares a standard image with a test image in order to find out the defects. This algorithm runs fast when defects in tracks are to be detected. This process is fast because it has only two types or levels of pixel values to compare, one for the tracks and the other for background. If there is an error then the difference operation detects that defect. The same process takes a lot of time to execute once we consider a mounted
PCB. This happens because there are now a lot of pixel values to be compared with and even though it gives a proper output but the processing time is very long. It takes approximately 2.20 minutes to give the output after simulation, which is quite a long time when we consider industry factors.

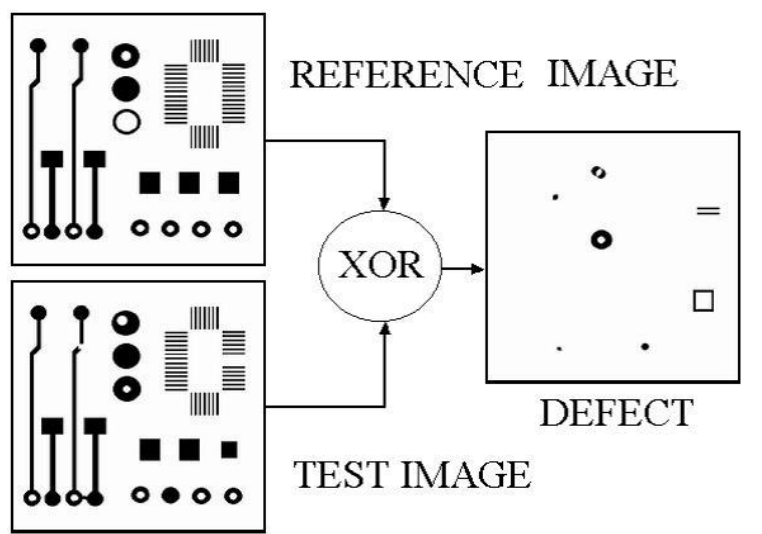

Fig 3. Subtraction Using XOR Operator

3.1.3 It is essential to see the performance of the inspection system in term of inspection time. The cost of processing depends on the size of the input image. When we consider only tracks, then the size of the image is small. But as soon as we consider a mounted PCB, sizes may vary in the range of $400 \times 400$ pixels. Hence Haar wavelet transform has been proposed many times to reduce the size of the image. Once the size of the image gets reduced, basic image subtraction operation can be performed. The coarse level processing is only applied for defects detection process. However, the defects localization should be highlighted on the original test image for better visual perception. Due to these results, the proposed algorithm is expected to detect several types of circuit printing defects such as breakout, short, pin hole, wrong size holes, open circuit, conductor too close, underetch, spurious copper, mouse-bite, excessive short, missing conductor, missing hole, spur and over-etch. This system reduces the processing time drastically. It takes approximately 3-4 seconds to give the output once wavelet transform is performed on the image.

But this algorithm too has its shortcomings. Wavelet transform compresses the image and because of it, certain important details of an image gets neglected. This may lead to discrepancies in the output as features missed in compression may result in defects after subtraction, which in reality may not be an actual defect.

3.1.4 Thresholding is an important technique for image segmentation that tries to identify and extract a target from its background on the basis of the distribution of grey levels or texture in image objects. Most thresholding techniques are based on the statistics of the one-dimensional (1D) [13] histogram of grey levels and on the two-dimensional (2D) cooccurrence matrix of an image. Locating the thresholds is done through parametric or nonparametric approaches. In parametric approaches, the grey level distribution of an object class leads to finding the thresholds. For instance, in Wang and Haralick's study [14], the pixels of an image are first classified as either edge non-edge pixels. According to their local neighborhoods, edge pixels are then classified as being relatively dark or relatively bright. Next, one histogram is obtained for those edge pixels which are dark and another for those edge pixels which are bright. The highest peaks of these two histograms are chosen as the thresholds. Moment 
preserving thresholding is a parametric method which segments the image based on the condition that the threshold image has the same moments as the original image [15]. In nonparametric approaches, the thresholds are obtained in an optimal manner according to some criteria. For instance, Otsu's method chooses the optimal thresholds by maximizing the between-class variance with an exhaustive search [16]. Otsu's method uses an exhaustive search to evaluate the criterion for maximizing the between-class variance. As the number in classes of an image increases, Otsu's method takes too much time to be practical for multi-level threshold selection. To determine the 1D threshold of an image efficiently, a modified between-class variance for Otsu's method is proposed. The recursive form of the proposed modified between-class variance will considerably decrease the computation of summing both the zeroth order moment and the first order moment of the probability density function. It is designed to replace the computation of the modified between-class variance for the 1D multilevel thresholding.

The above algorithm using thresholding and to be more specific using otsu's thresholding can differentiate between components and their defects, but if there is a large difference between the grey levels because of a variety of components present on the PCB, then after subtraction operation [Fig 4], a lot of artifacts get added to the image along with actual defects.

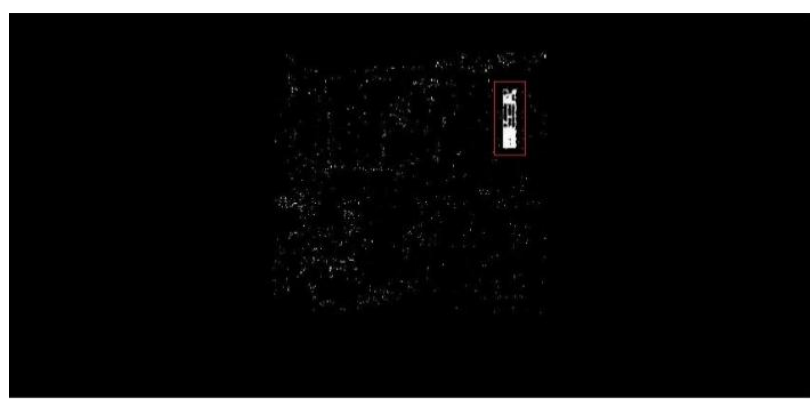

Fig 4. Artifacts included along with actual defects after Thresholding and Subtraction

3.1.5. The next algorithm proposes to train the system based on machine learning and use a mathematical model to detect the defects. Gaussian function is used to evaluate the system and then uses subtraction operation to show the defects. In this approach, specific alignment of the camera is used to capture the image and then using mean, standard variance and probability density functions, pixel values are estimated. Though the above algorithm trains the system for large number of data set and different types of errors, but still its time consuming and complex. Mathematical formulations itself make the system complex and it cannot be generalized for all PCBs. Also background learning is to be performed along with parameter estimation. Subtraction operation is performed at the end of the entire complex process.

\subsection{Optimal Approach}

To overcome the shortcomings of all previous algorithms, a better and faster process is proposed which provides both quantitative and qualitative results. The process starts with the capturing of the image of the PCB using a high resolution camera. While capturing of the image, proper care must be taken to check the alignment of the PCB as well as the camera. The captured image is then stored for further processing. The image captured is in the RGB (Red Green Blue) format. Next step of the process involves converting the coloured image into grey scale image. For a coloured image, there are different types of cones. Some are sensitive to red light, some to green light, and some to blue light. By emitting a controlled combination of these three basic colours (red, green and blue), and hence stimulate the three types of cones at will, one is able to generate almost any perceivable colour. Such coloured images are stored in an RGB format. In grey scale images, however, it cannot be differentiated how much of the different colours is emitted, it emits the same amount in each channel. What can be differentiated, is the total amount of emitted light for each pixel; little light gives dark pixels and much light is perceived as bright pixels. When converting an RGB image to grey scale, RGB values for each pixel is taken and converted into a single value, reflecting the brightness of that pixel. One such approach is to take the average of the contribution from each channel: $(\mathrm{R}+\mathrm{B}+\mathrm{C}) / 3$. Due to conversion of image each pixel gets stored as a byte ( 8 bits). The image gets pixel value in a range of ' 0 ' (black/dark) to '255' (white/bright). This makes it easy for further operations to be performed on the image.

The next step of the algorithm includes noise removal of the input image [Fig 5]. Various types of noise are present during capturing and transmission of the image. Depending upon the probability density functions some common type of noise present are Gaussian noise and salt and pepper noise. A special case is white Gaussian noise, in which the values at any pair of times are identically distributed and statistically independent (and hence uncorrelated). Principal sources of Gaussian noise in digital images arise during acquisition e.g. sensor noise caused by poor illumination and/or high temperature, and/or transmission e.g. electronic circuit noise.[17] In digital image processing Gaussian noise can be reduced using a spatial filter. The other noise usually present is the salt and pepper noise. It presents itself as sparsely occurring white and black pixels. An effective noise reduction method for this type of noise is a median filter [18]. In this algorithm, median filter is used. The averaging filter removes the noise by blurring it till is seen. But in this process it also blurs the edges of the image. As the averaging mask gets bigger the blurring of the image is more. As this will ruin the edges and important parts of the image, it is better to opt for median filter. Here in this process a nonlinear median filter is used. It is an order statistic filter. It is called as order statistic filter because the removal of noise depends upon the order in which pixels are present within the mask. Also median filter gives good results even if the amount of noise present in the image is large.

After noise removal, zooming operation is performed on the image. There may be many minute details on the PCB. So in order to avoid missing of any such feature, zooming is done. Zooming is performed either by replication or by interpolation. In replication we simply replicate each pixel and then replicate each row. No new data is added to the image. In interpolation, instead of replicating each pixel, average of the two adjacent pixels along the rows is taken and placed between the two pixels. The same operation is performed along the columns. The operation is done along the interlaced image. The zooming operation increases the clarity of small components and also at the same time increases the clarity of text on components, so that proper placement of components can be verified. Zooming can also help to detect small defects in tracks. After noise removal and zooming of the image, certain features of the PCB may still not be properly visible. Then laplacian operator is used to sharpen the image and simple averaging filter is used to smoothen the image, so that the image details are properly visible. 


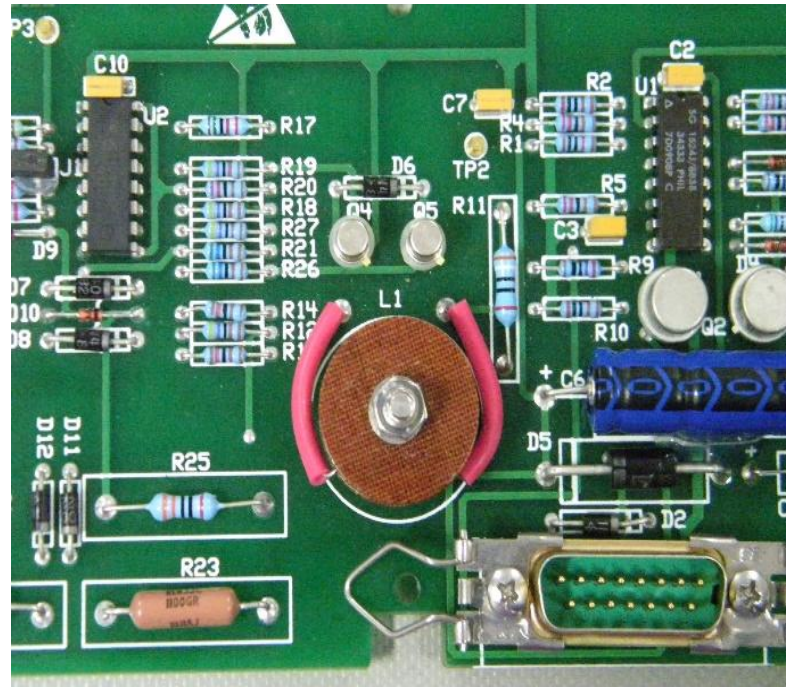

Fig 5. Input Image (Reference PCB)

After smoothening and sharpening of the image, histogram stretching and equalization is performed. Histogram of images provides a global description of the appearance of the image. The information obtained from histogram is enormous and hence histogram modelling is used at this step of process. Histogram represents the relative frequency of occurrence of the various grey levels in an image. Linear stretching is a good technique and the shape of histogram remains same. The value of pixels is simply magnified in order to make them visible. The pixel value of certain areas in an image may be very small after applying basic morphological operations. So histogram stretching can be used to magnify them in range with other values. Histogram equalization is used because after applying the image processing algorithms, the pixel value of respective components may vary over a wide range. So histogram equalization increases the value of small pixels and decreases the value of large pixels to get all values in a uniform range. This is also done with the image of the PCB in order to detect defects and normalize them to a common scale.

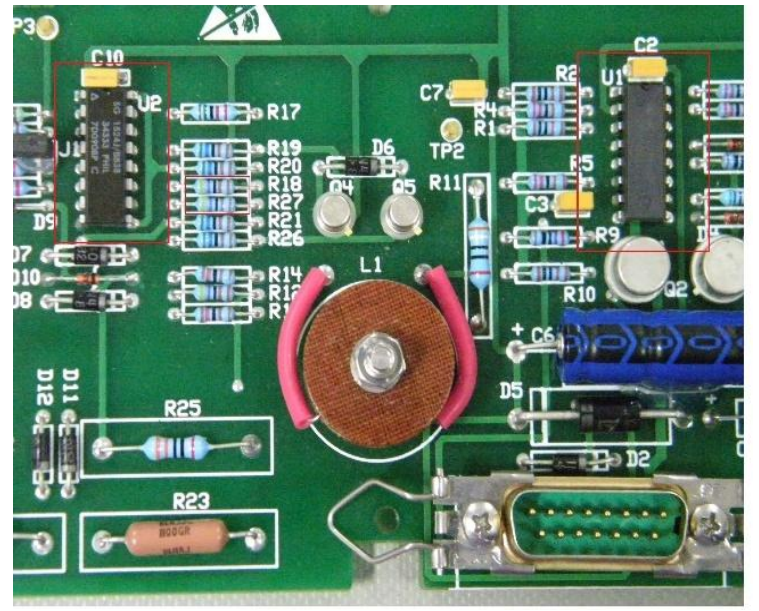

Fig 6. Defected Image

Once histogram stretching and equalization of the defected image [Fig 6] is performed, the next step in the flow is haar wavelet transform of the image. This step reduces the size of the input image by compressing it, in order to execute the further steps in a faster way. Proper care has to be taken in the given process so that specific details of the image are not lost during compression using wavelet transform. As it has been discussed earlier haar wavelet transform considerably increases the speed of subtraction of two images.

The final step in the algorithm is to subtract the image obtained after wavelet transform from the reference image. This gives the difference between both the images and the defect can be detected. It is faster in terms of computation speed and accuracy is also higher. The output thus obtained can be visualized [Fig 7].

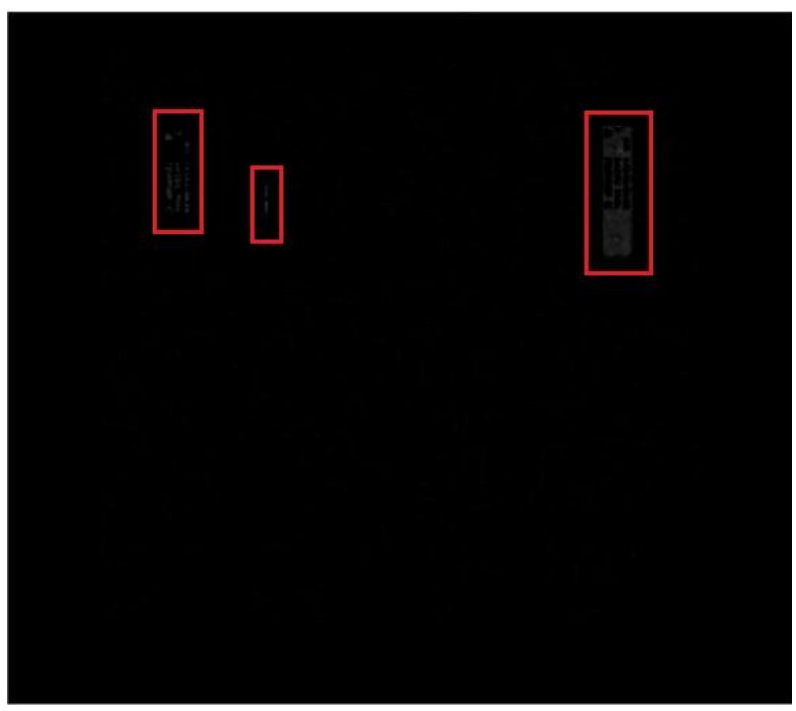

Fig 7. Subtracted Image Showing Defects

\section{RESULTS}

Once the algorithm was developed, it was implemented on sample PCBs to verify the results. The algorithm was tested on three mounted PCBs and three bare PCBs and the simulation time for each was calculated. The simulation time using MATLAB is tabulated in the table below to show the difference between simple subtraction operation of reference PCB and test PCB and subtraction after processing the PCB using proposed algorithm. (Table No.1).

\section{Table No.1: Simulation Time with different Sample PCBs}

\begin{tabular}{|l|l|l|l|}
\hline $\begin{array}{l}\text { Sample } \\
\text { number }\end{array}$ & $\begin{array}{c}\text { Type of } \\
\text { sample } \\
\text { subtraction } \\
\text { (in } \\
\text { seconds) }\end{array}$ & $\begin{array}{c}\text { Simple } \\
\text { with wavelet } \\
\text { transform } \\
\text { (in seconds) }\end{array}$ \\
\hline 1 & $\begin{array}{l}\text { mounted } \\
\text { PCB 1 }\end{array}$ & 1.518985 & 0.304917 \\
\hline 2 & $\begin{array}{l}\text { mounted } \\
\text { PCB 2 }\end{array}$ & 1.275309 & 0.382957 \\
\hline 3 & $\begin{array}{l}\text { mounted } \\
\text { PCB 3 }\end{array}$ & 0.372908 & 0.343498 \\
\hline 4 & bare PCB 1 & 0.847678 & 0.362650 \\
\hline 6 & bare PCB 2 & 0.607978 & 0.360874 \\
\hline
\end{tabular}

Based on the table above, the difference between simulation time of simple subtraction and using the proposed algorithm with wavelet transform can be observed. There is drastic reduction in simulation time which is very beneficial in terms of industry factors. A graphical representation of the above table is shown below for a better interpretation of the data. 


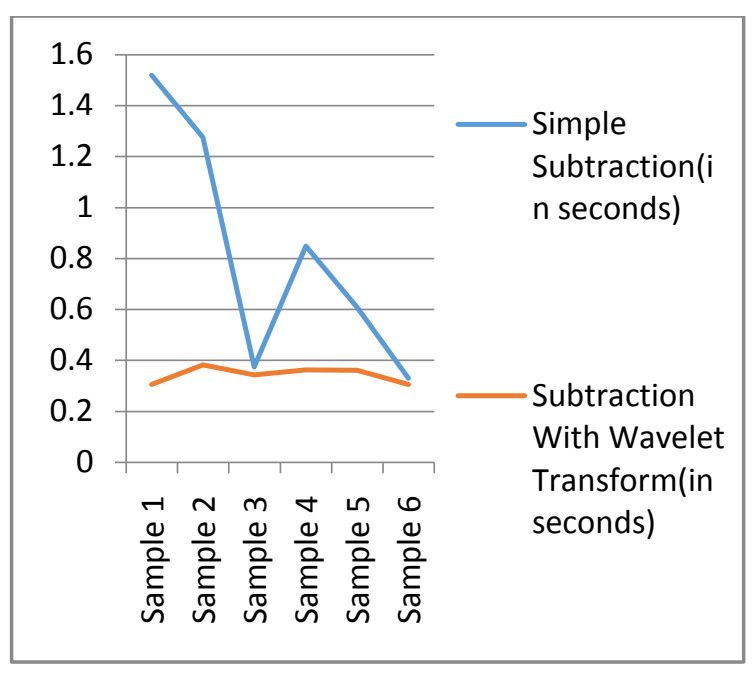

Fig 8. Graphical Interpretation of Simulation Time

Considering an example, the simulation time for a mounted PCB for sample 1 took 1.518985 seconds to execute and it took 0.304917 seconds to process the same image using the proposed algorithm and produce suitable outputs. Depending on the complexity of the PCB and the amount of components mounted on the PCB, the execution time will vary and accordingly the difference between both the processes. For a bare PCB, the difference between simple subtraction and subtraction through above algorithm may be less, as it is evident from the above data, but when it comes to industrial applications, the complexity of PCBs has increased both in terms of component density as well as layers available in one $\mathrm{PCB}$, so the proposed algorithm can be more helpful. Also the number and types of defects detected using the given algorithm is more than previous algorithms.

\section{CONCLUSION}

Automatic PCB inspection is needed to inspect the PCB for defect, anomalies and fault. Among the variety of algorithms, the image difference operation has been emphasized more in order to get better results. The system proposed is not a generalized system but a dedicated system for a particular PCB and can be optimized to a level. This has also been noted that the system cannot be generalized as each PCB inspection is different from its assembly point of view. Hence it is noted that different operations are found suitable for different PCBs according to its features. The drawback of different proposed algorithms have been studied and accordingly an optimal approach is used to minimise the shortcomings and increase the operation speed. The major limitation of existing inspection systems is that all the algorithms need a special hardware platform to achieve the desired real-time speeds. This makes the systems extremely expensive. Any improvements in speeding up the computation process algorithmically could reduce the cost of these systems drastically. However, they remain a better option than increasingly error prone, and slow manual human inspection.

The proposed algorithm is faster as compared to previous algorithms and can also detect a diverse range of defects. It has been able to eradicate the problems faced with simple morphological operations, subtraction, wavelet transform and thresholding operation.

There may be certain real time challenges while capturing of the image and hence those shortcomings are to be overcome. The following algorithm was applied on 4 different types of bare PCBs and it was found to be almost $90 \%$ accurate. Also the same process was applied on 5 different types of mounted PCBs with $80-85 \%$ accuracy.

\section{ACKNOWLEDGMENTS}

We are thankful to Head of the Electronics Engineering Department, Vishwakarma Institute of Technology, Pune for his valuable guidance and continuous support in development of this concept.

\section{REFERENCES}

[1] F. Moganti, F. Ercal, C. H. Dagli, S.Tsunekawa. "Automatic PCB inspection algorithms:A survey, Computer Vision and Image Understanding", Vol. 63,No. 2, pp. 287-313, 1996.

[2] Wen-Yen, J. Mao-Jiun, J.Wang, L. Chih-Ming. "Automated inspection of printed circuit board through machine vision, Computers in Industry", Vol.28, Issue 2, pp. 103-111, 1996.

[3] J. H. Koo, S. I. Yoo. "A structural matching for twodimensional visual pattern inspection, IEEE International Conference on Systems, Man, and Cybernatics", Vol. 5, pp. 4429-4434, 1998.

[4] M. H. Tatibana, R. de A. Lotufo. "Novel automatic PCB inspection technique based on connectivity,Proceedings of Brazilian Symposium on Computer Vision and Image Pro cessing", pp. 187-194, 1997.

[5] M.Ouslim, K. M. Curtis."PCB inspection based on a variant of the N-tuple technique, Fifth International Conference on Image Processing and its Applications",pp. 677-681,1995.

[6] F.Ercal, F. Bunyak, H. Feng, L. Zheng. "A fast modular RLE-based inspection scheme for PCBs,Proceedings of SPIE Architectures, Networks and Intelligent Systems for Manu facturing Integration", Vol.3203, pp. 45-59, 1997.

[7] F. Ercal, F. Bunyak, H. Feng.” Context-sensitive filtering in RLE for PCB inspection,Proceedings of SPIE Intelligent Systems and Advanced Manufacturing”, Vol. 3517,1998

[8] F. Ercal, M. Allen, H. Feng. "Proof of correctness and performance analysis of a systolic image difference algorithm for RLE-compressed images, IEEE Transaction on Parallel and Distributed Computing Systems", Vol. 11, No. 5, pp. 433-443, 2000.

[9] S. H. Oguz, L. Onural.” An automated system for designrule-based visual inspection of printed circuit boards, Proceedings of the IEEE International Conference on Robotics and Automation”, pp.2696-2701, 1991.

[10] A. M. Darwish, A. K. Jain.” A rule based approach for visual pattern inspection, IEEE Transaction of Pattern Analysis and Machine Intelligence", Vol.PAMI-10, No. 1, pp. 56-58, 1988.

[11] Y. Qin-Zhong, P. E. Danielson. "Inspection of printed circuit boards by connectivity preserving shrinking, IEEE Transaction on Pattern Analysis and Machine Intelligence", Vol. PAMI-10, No. 5.

[12] Zuwairie Ibrahim, Syed Abdul Rahman Al-Attas and Zulfakar Aspar," Model-based PCB Inspection Technique Using Wavelet Transform", The 4th Asian Control Conference, Singapore, pp.55-58, 2002. 
[13] Hamed Kiani, Reza Safabakhsh, Ehsan Khadangi. "Fast Recursive Segmentation Algorithm Based on Kapur's Entropy".

[14] Sanveer Singh, Manu Bharti," Image Processing Based Automatic Visual Inspection System for PCBs", IOSR Journal of Engineering (IOSRJEN) ISSN: 2250-3021 Volume 2, Issue 6 ,PP 1451-1455, June 2012.

[15] D. M. Tsai and Y. H. Chen, "A fast histogram-clustering approach for multilevel thresholding," Pattern Recognition Letters, Vol. 13, No. 4, 1992, pp. 245-252.
[16] J. C.Yen, F. J.Chang, and S. Chang, "A new criterion for automatic multilevel thresholding,"IEEE Transactions on Image Processing, Vol. 4, No. 3, 1995, pp. 370-378.

[17] Dr. Philippe Cattin (2012-04-24). "Image Restoration: Introduction to Signal and Image Processing". MIAC, University of Basel. Retrieved 11 October 2013.

[18] Jayaraman et al. (2009). Digital Image Processing. Tata McGraw Hill Education. p. 272. 